\title{
EVOLUÇÃO DA PRODUÇÃO CIENTíFICA EM EMPREENDEDORISMO RURAL: UM ESTUDO BIBLIOMÉTRICO
}

Evelyn Bernardo ${ }^{1}$

Heidy Rodriguez Ramos ${ }^{2}$

Leonardo Vils ${ }^{3}$

\footnotetext{
${ }^{1}$ Universidade Nove de Julho (Uninove) / Doutoranda do Programa de Pós-graduação em Administração (PPGA)

${ }^{2}$ Universidade Nove de Julho (Uninove)/ Professora no Mestrado Profissional em Administração - Gestão Ambiental e Sustentabilidade (GeAS), Mestrado em Cidades Inteligentes e Sustentáveis (CIS) e Programa de Pós-graduação em Administração (PPGA).

${ }^{3}$ Universidade Nove de Julho (Uninove)/ Professor no Mestrado Profissional em Administração - Gestão Ambiental e Sustentabilidade (GeAS)
} 


\section{EVOLUÇÃO DA PRODUÇÃO CIENTÍFICA EM EMPREENDEDORISMO RURAL: UM ESTUDO BIBLIOMÉTRICO}

O artigo tem como objetivo realizar análise do panorama das publicações sobre empreendedorismo rural por meio de um estudo bibliométrico. Para tanto, o estudo realizou a análise de 417 artigos publicados na plataforma Web of Science ligados ao tópico "empreendedorismo rural" na língua inglesa, traçando a evolução das publicações por meio de análises de citações e de co-citações em todos os artigos publicados na plataforma. Esse método possibilitou a identificação das obras mais influentes com a finalidade de entendimento dos laços intelectuais e evolução de pesquisa sobre o tema proposto ao longo do tempo. Os resultados obtidos possibilitaram a identificação dos autores mais citados, formação de quatro cluster de autores que trabalham com o tema empreendedorismo rural, teorias que apontam que o campo de pesquisa está em ascensão e que o uso do termo "empreendedorismo rural" vem crescendo no meio acadêmico e se consolidando.

\section{Introdução}

O empreendedorismo é determinado como um dos principais propulsores do crescimento econômico, por meio da criação de emprego e renda para uma população. Relação essa, entre desenvolvimento e empreendedorismo que foi inicialmente proposta por Schumpeter em 1934. E que também pode ser observado como um fenômeno que cresce em diversas esferas, seja no empreendedor como indivíduo ou de um processo de desenvolvimento empreendedor de uma empresa (Venkataraman, 1997).

No meio rural, o Empreendedorismo Rural, não é propriamente um terno novo, mas seu uso é recente e com larga concentração nas políticas de governo, nos movimentos sociais e nos meios acadêmicos. No Brasil, por meio do poder público o desenvolvimento do setor agrícola surge na implantação do Programa de Fortalecimento da Agricultura Familiar (PRONAF) (Brasil, 1996), ou quando cria a Lei 11.326/2006, que considera Agricultor Familiar e Empreendedor Familiar Rural como aquele que pratica atividades no meio rural (Brasil, 2006).

Nos movimentos sociais, Venkataraman, Vermeulen, Raaijmakers \& Mair (2016), analisaram as atividades da Assistência Profissional para a Ação de Desenvolvimento (PRADAN) - uma ONG que trabalha em áreas rurais pobres na Índia para promover o desenvolvimento social utilizando lógicas de mercado.

Nos meios acadêmicos o Empreendedorismo Rural é visto de várias maneiras, descrevendo principalmente como empreendedores que praticam atividades agrícolas de cultivo ou de criação de animais que gerem fonte de renda em uma perspectiva de gestão e desenvolvimento do setor agrícola (Henry \& McElwee, 2014; McElwee, 2006). 
Como podemos observar, o desenvolvimento no meio rural por meio do empreendedorismo vem acontecendo nas três esferas, como ações em políticas de governo, nos movimentos sociais, por meio do fortalecimento de iniciativas sociais e no meio acadêmico por meio de estudos de empreendedorismo no meio rural.

Para entendermos essa possível evolução em pesquisas no meio acadêmico, o estudo tem como objetivo realizar análise das publicações sobre Empreendedorismo Rural por meio de um estudo bibliométrico. Para tanto, o estudo realizou a análise de 417 artigos publicados na plataforma Web of Science ligados ao tópico empreendedorismo rural na língua inglesa, traçando a evolução das publicações por meio de análises de citações e de co-citações em todos os artigos publicados na plataforma.

\section{Referencial Teórico}

A Diretriz teórica da pesquisa fundamentou-se na contextualização da "Agricultura familiar" e na diversidade de estudos que enfocam este conceito no Brasil, sua possível evolução e os principais conceitos do empreendedor no meio rural. Neste sentido, o mapeamento da produção científica, auxilia no reconhecimento dos principais autores e obras que compõe o campo teórico.

\subsection{O Meio Rural e o Agricultura de Origem Familiar}

A agricultura no meio rural é predominantemente de agricultores da agricultura familiar. Um agricultor familiar, ao mesmo tempo que é dono da terra, produz o seu próprio alimento e comercializa o excedente, ou seja, trabalho intimamente ligado a família (Lamarche, 1993; Wanderley, 1998). No meio rural, o agricultor familiar também é visto como um ator social do mundo moderno, por sua capacidade de adaptação aos novos contextos econômicos e sociais (Wanderley, 2003).

$\mathrm{Na}$ tentativa de promover resposta e soluções aos desafios do mundo moderno, começam a surgir mudanças nos novos modelos de organizações. Essas mudanças dizem respeito principalmente às reformas políticas, como a criação da Lei no. 11.326, que considera Agricultor Familiar e Empreendedor Familiar Rural como aquele que realiza atividades no meio rural (Brasil, 2006).

Também é possível observar as mudanças no entendimento do termo Agricultor Familiar, que nasce com origem no núcleo familiar, mas que ao longo do tempo começa buscar por desenvolvimento e habilidades, estimulando assim a criação de redes e organizações, pequenas empresas comerciais ou cooperativas agrícolas - processo de fortalecimento da Agricultura Familiar (Abramovay, Magalhães, \& Schroder, 2010; Veiga, 2001). Processo de desenvolvimento que também é debatido por muitos pesquisadores ( Van 
der Ploeg, Renting, Brunori, Knickel, Mannion, Marsden, de Roest, Sevilla-Guzman \& Ventura, 2000).

Neste processo de mudança, o empreendedorismo no meio rural é visto como forma de desenvolvimento, por transformar a realidade econômica rural, ao mesmo tempo que melhora a criação de valor, oportunidade e emprego.

Já em um ambiente empresarial, o termo empreendedor vem do empreendedorismo que é voltado para o desenvolvimento de competências e habilidades relacionadas a criação de um projeto (técnico, científico, empresarial). Que em sua visão mais clássica do sentido de ser empreendedor de Shumpeter (1950), se dá aos aspectos relacionados às características do indivíduo, aos seus valores e ao modo de pensar e agir.

\subsection{Empreendedorismo e o Empreendedorismo rural}

Joseph Schumpeter contribuiu de forma significativa para a Teoria do Empreendedorismo por meio da Teoria do Desenvolvimento Econômico de 1934, com a definição do empresário como inovador, aquele que realiza novas combinações, como: 1) introdução de um novo bem; 2) introdução de um novo método de produção; 3) abrir um novo mercado; 4) utilização de uma nova fonte de abastecimento; 5) criação de algumas novas formas organizacionais em uma indústria (Schumpeter, 1982).

No meio rural, a criação de um novo produto, mercado ou tecnologia também pode ser enfatizado por aspectos de inovação, como: o agro turismo, inovações tecnológicas, de processos, organizacional (por exemplo, estruturas de cooperação entre atores, como é o caso da iniciativa de redes de negócios empreendedores) e inovações em atitudes através de uma cultura de cooperação (Esparcia, Escribano \& Serrano, 2015; Pato \& Teixeira, 2016).

Um empreendedor bem-sucedido está envolvido em esforços econômicos ativos, dinâmicos e competitivos, em uma busca contínua da oportunidade. Os espaços rurais também oferecem uma série de oportunidades e possibilidades para os empreendedores em potenciais. $\mathrm{Na}$ literatura de desenvolvimento rural, o conceito empreendedorismo tem sido utilizado como perspectiva de crescimento, seja enfatizando a industrialização da produção agrícola ou por meio de sua produção em grande escala, maximização de lucros e tomada de risco (Niska, Vesala, \& Vesala, 2012).

Na concepção inicial do empreendedorismo de Wortman (1990), o empreendedorismo rural é definido como "a criação de uma nova organização que introduz um novo produto, desenvolvimento ou criação de um novo mercado, ou utilização de uma nova tecnologia em um ambiente rural" (Wortman, 1990, página 330).

O desenvolvimento do agricultor pode ser analisado de diferentes formas, Smith \& McElwee (2015), exploram o desenvolvimento de habilidades empresariais em agricultores, destacando que a "agricultura familiar é a espinha dorsal do modelo europeu de agricultura". 


\section{Métodos e técnicas de pesquisa}

O presente estudo tem caráter teórico-descritivo, abordando o debate conceitual dos principais termos utilizados para caracterizar iniciativas de estudo no campo do empreendedorismo e descrevendo as características de uma determinada população de empreendedores que estão presentes no meio rural ou agrícola.

Um estudo bibliométrico é uma técnica quantitativa, que tem como propósito a medição dos índices de produção e a disseminação do conhecimento científico. Conhecimento que envolve padrões e modelos matemáticos para medir processos, usando seus resultados para elaborar previsões e apoio a tomadas de decisão. Aspectos quantitativos da produção, da disseminação e do uso da informação registrada (Tague-Sutcliffe, 1992).

No decorrer da revisão da literatura, alguns estudos bibliográficos foram localizados e considerados no estudo, são eles: o estudo de McElwee (2006), que forneceu uma descrição do empreendedorismo agrícola, indicando como pesquisas futuras, estudos que analisem habilidades empresariais dos agricultores. A pesquisa de Pato \& Teixeira (2016), que analisou 181 artigos em empreendedorismo rural em periódicos publicados na base Scopus, concentrando apenas em países desenvolvidos e excluindo a produção científica publicada em outras fontes.

\subsection{Coleta de dados}

A coleta de dados foi realizada por meio da plataforma Web of Science da Thompson Reuters, plataforma também utilizada por diversas pesquisas anteriores (Ferreira, Reis, \& Miranda, 2015; L. M. Zanin \& Silva, 2015).

Inicialmente, na plataforma, no campo de busca foram utilizados diversos termos para identificar o campo de estudo e posteriormente abrangendo e limitando a pesquisa, utilizando os critérios de busca booleana como: or, and, not, entre outros. Os termos utilizados foram "rural entrepreneur***", "agricultural business entrepreneur***", "agricultural family entrepreneur***", "new agricultural enterprises", "agricultural business" or "rural entrepreneurs". Todos esses termos foram utilizados para análise do campo para seleção de artigos de empreendedorismo no meio rural, visto que utilizamos sinônimos para abordar o um assunto em específico. Filtro para seleção somente de artigos foram realizados, excluindo livros e resumos da seleção. Neste processo os artigos mais relevantes foram selecionados, totalizando 417 artigos para leitura prévia dos títulos, resumos e palavras-chave.

\section{Análise e Interpretação dos dados}

A análise dos artigos 417 estudos publicados em periódicos acadêmicos, foram realizadas utilizando softwares para tratamento das informações. O software VOSviewer, permitiu a organização de grupos (cluster) que reúnem co-citações, em uma perspectiva qualitativa e para criação de mapas de relacionamentos (redes) e clusters, em publicações de 
coautoria ou co-ocorrência, seja de autores, journals ou de palavras chaves que se destacam no texto. O Software também é utilizado no meio acadêmico em estudo bibliométricos e em diversos estudos no campo do empreendedorismo (Barreto \& Nassif, 2014; Van Eck \& Waltman, 2014; Zanin \& Silva, 2015).

Por meio do Software Bibexcel, foi possível realizar o agrupamento de citações de todas as referências, as referências foram ordenadas seguindo o critério "das mais citadas", para "as de menor citação", selecionando e formando uma base de dados com um número de 70 autores, número suficiente para a realização da análise de citação e cocitação (Serra, F. R., Ferreira, M. P., de Almeida, M. I. R., \& de Souza Vanz, 2012). Utilizando o software BibExcel, foi possível analisar qualitativamente os dados, fornecendo informações para Análise Fatorial Exploratória (AFE) com o uso do software estatística SPSS.

Com o uso do software IBM-SPSS v.22, foi possível realizar a AFE, análise realizada por meio do Método de Componentes Principais (MCP) com rotação Varimax, que visa a redução de um grupo de variáveis em um conjunto menor de acordo com suas semelhanças (Hair, Black, Babin, Anderson \& Tatham, 2009; Pinto, Guerrazzi, Serra, \& Kniess, 2016).

Nesta seção serão apresentados os principais resultados encontrados na busca de pesquisa do ISI Web of Science, por meio da análise da produção científica em empreendedorismo no meio rural.

\subsection{Contextualização da produção científica}

A contextualização da produção científica é importante para que por meio dela seja possível a compreensão do processo de construção do tema ao longo do tempo. Para a realização desta análise, a coleta de dados realizada na plataforma Web of Science foram selecionados e transferidos para a lista marcada gerando um arquivo em formato Excel. A Figura 1, apresenta essa evolução por ano, no período de 1972 a 2017.

\section{Figura 1 - Evolução da produção científica - Total de publicações ano}

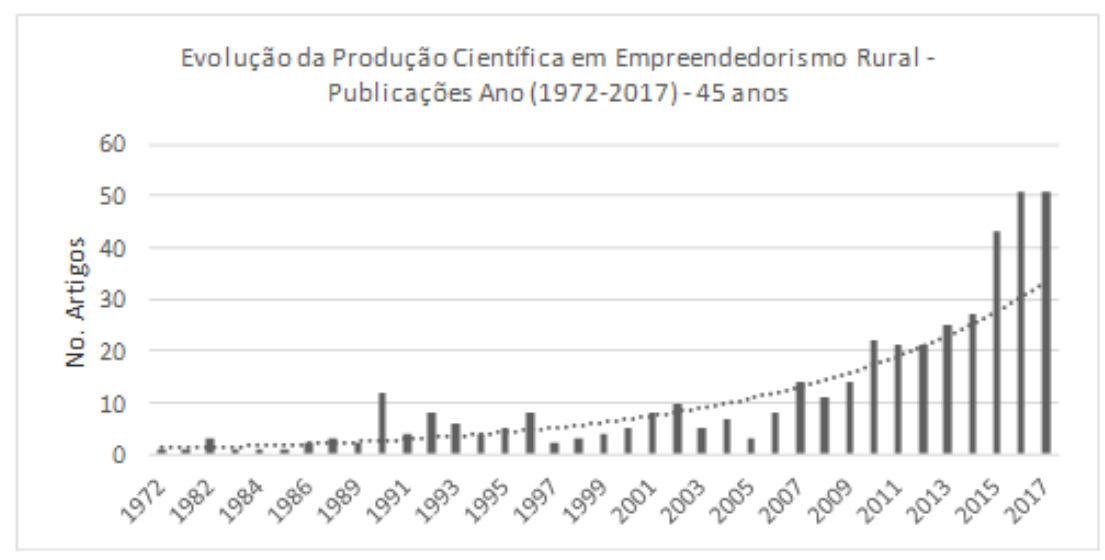

Fonte: Dados do ISI Web of Science (2017) 
No período analisado, é possível observar que mesmo o termo "Empreendedorismo Rural" não seja recente, seu uso manteve-se crescendo com picos de altos e baixos até 2011, possivelmente indicando uma tendência e consolidação do tema. Outra hipótese para esse aumento de publicações de 2014 para 2015 é que em 2014 a Organização das Nações Unidas (ONU), elegeu o tema "agricultura familiar" como tema do ano (2014) para 193 países membros. Eleição que aconteceu em dezembro deste ano na Assembleia Geral da ONU (FAO, 2014). Na Tabela 1, temos o ranking por periódicos que na amostra aparecem com maiores publicações no tema de pesquisa em "Empreendedorismo Rural" (ER).

Tabela 1 - Ranking dos periódicos e Fator de Impacto das revistas

\begin{tabular}{|cllrcr|}
\hline Classificação & \multicolumn{1}{c}{ Título do periódico } & $\begin{array}{c}\text { No. } \\
\text { Artigos em } \\
\text { E.R }\end{array}$ & $\begin{array}{c}\text { Citações } \\
\text { impacto } \\
\text { do }\end{array}$ & $\begin{array}{c}\text { Ponturação } \\
\text { Eigenfactor } \\
\text { periódico }\end{array}$ \\
\hline $\mathbf{1}$ & Journal Of Rural Studies & 25 & 3,398 & 2.380 & 0.001640 \\
\hline $\mathbf{2}$ & Entrepreneurship And Regional Development & 19 & 1,893 & 1.776 & 0.001790 \\
\hline $\mathbf{3}$ & American Journal of Agricultural Economics & 15 & 5,939 & 1.829 & 0.006560 \\
\hline $\mathbf{4}$ & Sociologia Ruralis & 11 & 1,466 & 1.698 & 0.001040 \\
\hline $\mathbf{5}$ & World Development & 11 & 12,778 & 2.848 & 0.019910 \\
\hline $\mathbf{6}$ & Sociologia Ruralis & 10 & 1,466 & 1.698 & 0.001040 \\
\hline $\mathbf{7}$ & European Planning Studies & 10 & 2,296 & 1.332 & 0.003270 \\
\hline $\mathbf{8}$ & Rural Sociology & 10 & 1,318 & 1.718 & 0.000980 \\
\hline $\mathbf{9}$ & Agriculture and Human Values & 10 & 1,742 & 2.337 & 0.002470 \\
\hline $\mathbf{1 0}$ & Regional Studies & 8 & 5,174 & 2.780 & 0.008060 \\
\hline $\mathbf{1 1}$ & Sustainability & 8 & 4,488 & 1.789 & 0.009090 \\
\hline $\mathbf{1 2}$ & Agricultural Systems & 8 & 4,495 & 2.571 & 0.005550 \\
\hline $\mathbf{1 3}$ & Agricultural Economics & 7 & 2,411 & 1.758 & 0.003300 \\
\hline $\mathbf{1 4}$ & International Journal of Agricultural Sustainability & 7 & 614 & 1.780 & 0.001110 \\
\hline $\mathbf{1 5}$ & Environmental Management & 7 & 8,436 & 1.878 & 0.008410 \\
\hline & & Total & 166 & & \\
\hline
\end{tabular}

Fonte: Elaborado pelos autores com os dados coletados da ISI Web of Science

Nesta análise foi considerada as informações obtidas do Journal Citation Reports (JCR) - 2016, indexado em 2017 nas publicações do Institute for Scientific Information (ISI), editada pela Thomson Reuters e indexada na Web of Science. No JCR, é possível identificar quadros qualitativos que cobrem as áreas distintas do conhecimento científico. A base apresenta dados qualitativos dos periódicos e realiza a avalição e comparação de periódicos por meio da acumulação e tabulação de contagens de citações e artigos de grande parte das especialidades e campos da ciência. O Fator de Impacto é o número médio de citações de artigos científicos publicados em determinado periódico. E o Eigenfactor é um índice que calcula a importância total de um periódico para a comunidade científica, não apenas a quantidade de citações mais a qualidade das mesmas.

Nesta análise foi possível identificar dentro da amostra, os periódicos que mais publicam sobre o tema "Empreendedorismo Rural", em primeira colocação temos o Journal 
Of Rural Studies, periódico que publica avanços na compreensão e análise das sociedades rurais com cobertura que tem alcance global.

\subsection{Análise das Citações}

$\mathrm{Na}$ tabela 2, pode ser observado o crescimento no número de citações que aparecem no período de (2001-2017). Nos anos iniciais (1980 a 2000), poucas publicações foram realizadas, tais publicações correspondem aos 30 autores que mais publicam sobre o tema.

Tabela 2 - Número de artigos mais citados - por autor e período (ano) de citação.

\begin{tabular}{|c|c|c|c|c|c|}
\hline No & Documentos Citados & $\begin{array}{l}1980- \\
2000\end{array}$ & $\begin{array}{l}2001- \\
2017\end{array}$ & \begin{tabular}{|l|} 
Total \\
de \\
citações \\
\end{tabular} & $\begin{array}{l}\text { Média } \\
\text { por } \\
\text { ano }\end{array}$ \\
\hline 1 & $\begin{array}{l}\text { Jack, S. L., \& Anderson, A. R. (2002). The effects of embeddedness on the entrepreneurial } \\
\text { process. }\end{array}$ & 0 & 346 & 346 & 20,35 \\
\hline 2 & Martinot et al. (2002). Renewable energy markets in developing countries. & 0 & 152 & 152 & 8,94 \\
\hline 3 & Gasson et al. (1988). The Farm as a Family Business - A Review. & 44 & 86 & 130 & 4,19 \\
\hline 4 & $\begin{array}{l}\text { Barbieri et al. (2009). Why is diversification an attractive farm adjustment strategy? Insights } \\
\text { from Texas farmers and ranchers }\end{array}$ & 0 & 93 & 93 & 9,30 \\
\hline 5 & $\begin{array}{l}\text { Marsden et al. (2002). The social management of rural nature: understanding agrarian-based } \\
\text { rural development. }\end{array}$ & 0 & 93 & 93 & 5,47 \\
\hline 6 & $\begin{array}{l}\text { Kajanus et al. (2004). The use of value focused thinking and the A'Wot hybrid method in } \\
\text { tourism management. }\end{array}$ & 0 & 92 & 92 & 6,13 \\
\hline 7 & $\begin{array}{l}\text { Vaillant et al. (2007). Do different institutional frameworks condition the influence of local } \\
\text { fear of failure and entrepreneurial examples over entrepreneurial activity? }\end{array}$ & 0 & 87 & 87 & 7,25 \\
\hline 8 & $\begin{array}{l}\text { Bergevoet et al. (2004). Entrepreneurial behaviour of Dutch dairy farmers under a milk quota } \\
\text { system: goals, objectives and attitudes. }\end{array}$ & 0 & 85 & 85 & 5,67 \\
\hline 9 & $\begin{array}{l}\text { North \& Smallbone (2006). Developing entrepreneurship and enterprise in Europe's } \\
\text { peripheral rural areas: Some issues facing policy-makers. }\end{array}$ & 0 & 69 & 69 & 5,31 \\
\hline 10 & Lordkipanidze et al. (2005). The entrepreneurship factor in sustainable tourism development. & 0 & 66 & 66 & 4,71 \\
\hline 11 & $\begin{array}{l}\text { Meccheri et al (2006). Rural entrepreneurs and institutional assistance: an empirical study } \\
\text { from mountainous Italy. }\end{array}$ & 0 & 58 & 58 & 4,46 \\
\hline 12 & $\begin{array}{l}\text { Kalantaridis \& Bika (2006)A. In-migrant entrepreneurship in rural England: beyond local } \\
\text { embeddedness. }\end{array}$ & 0 & 57 & 57 & 4,38 \\
\hline 13 & $\begin{array}{l}\text { Cajaiba-Santana, Giovany (2014). Social innovation: Moving the field forward. A conceptual } \\
\text { framework. }\end{array}$ & 0 & 56 & 56 & 11,2 \\
\hline 14 & $\begin{array}{l}\text { Lafuente et al. 2007. Regional differences in the influence of role models: Comparing the } \\
\text { entrepreneurial process of rural Catalonia. }\end{array}$ & 0 & 56 & 56 & 4,67 \\
\hline 15 & $\begin{array}{l}\text { Vanderploeg, JD (1993). Rural Sociology and The New Agrarian Question - A Perspective from } \\
\text { the Netherlands. }\end{array}$ & 15 & 41 & 56 & 2,15 \\
\hline 16 & $\begin{array}{l}\text { Barbieri \& Mshenga (2008). The role of the firm and owner characteristics on the performance } \\
\text { of agritourism farms }\end{array}$ & 0 & 55 & 55 & 5,00 \\
\hline 17 & $\begin{array}{l}\text { Kalantaridis \& Bika (2006)B. Local embeddedness and rural entrepreneurship: case-study } \\
\text { evidence from Cumbria, England. }\end{array}$ & 0 & 54 & 54 & 4,15 \\
\hline 18 & $\begin{array}{l}\text { Bock, BB (2004). Fitting in and multi-tasking: Dutch farm women's strategies in rural } \\
\text { entrepreneurship. }\end{array}$ & 0 & 52 & 52 & 3,47 \\
\hline 19 & $\begin{array}{l}\text { Stone \& Stubbs (2007). Enterprising expatriates: Lifestyle migration and entrepreneurship in } \\
\text { rural southern Europe. }\end{array}$ & 0 & 45 & 45 & 3,75 \\
\hline 20 & $\begin{array}{l}\text { Vik \& McElwee (2011). Diversification and the Entrepreneurial Motivations of Farmers in } \\
\text { Norway. }\end{array}$ & 0 & 43 & 43 & 5,38 \\
\hline 21 & $\begin{array}{l}\text { Sturgeon, Janet C. (2010). Governing minorities and development in Xishuangbanna, China: } \\
\text { Akha and Dai rubber farmers as entrepreneurs. }\end{array}$ & 0 & 39 & 39 & 4,33 \\
\hline 22 & $\begin{array}{l}\text { Henderson \& Weiler (2010). Entrepreneurs and Job Growth: Probing the Boundaries of Time } \\
\text { and Space. }\end{array}$ & 0 & 37 & 37 & 4,11 \\
\hline
\end{tabular}




\begin{tabular}{|l|l|l|l|l|l|}
$\mathbf{2 3}$ & Demurger \& Xu (2011). Return Migrants: The Rise of New Entrepreneurs in Rural China. & 0 & 33 & $\mathbf{3 3}$ & 4,13 \\
\hline $\mathbf{2 4}$ & $\begin{array}{l}\text { Driga et al. (2009). Reasons for the Relatively Lower Entrepreneurial Activity Levels of Rural } \\
\text { Women in Spain. }\end{array}$ & 0 & 33 & $\mathbf{3 3}$ & 3,30 \\
\hline $\mathbf{2 5}$ & $\begin{array}{l}\text { Chang \& Macmillan (1991). A Review of Entrepreneurial Development in the Peoples- } \\
\text { Republic-Of-China. }\end{array}$ & 8 & 25 & $\mathbf{3 3}$ & 1,18 \\
\hline $\mathbf{2 6}$ & $\begin{array}{l}\text { Anthopoulou, Theodosia (2010). Rural women in local agrofood production: Between } \\
\text { entrepreneurial initiatives and family strategies. A case study in Greece. }\end{array}$ & 0 & 31 & $\mathbf{3 1}$ & 3,44 \\
\hline $\mathbf{2 7}$ & Satya et al. (2010). Bamboo shoot processing: food quality and safety aspect. & 0 & 31 & $\mathbf{3 1}$ & 3,44 \\
\hline $\mathbf{2 8}$ & $\begin{array}{l}\text { lark, Julian (2009). Entrepreneurship and diversification on English farms: Identifying } \\
\text { business enterprise characteristics and change processes. }\end{array}$ & 0 & 31 & $\mathbf{3 1}$ & 3,10 \\
\hline $\mathbf{2 9}$ & $\begin{array}{l}\text { Ma, ZD (2002). Social-capital mobilization and income returns to entrepreneurship: the case } \\
\text { of return migration in rural China. }\end{array}$ & 0 & 31 & $\mathbf{3 1}$ & 1,82 \\
\hline $\mathbf{3 0}$ & $\begin{array}{l}\text { Herslund, Lise (2012). The Rural Creative Class: Counterurbanisation and Entrepreneurship in } \\
\text { the Danish Countryside. }\end{array}$ & 0 & 30 & $\mathbf{3 0}$ & 4,29 \\
\hline
\end{tabular}

Fonte: Dados do ISI Web of Science com Bibexcel.

Na Tabela 2, também é possível observar os autores mais citados dentro da amostra. Na primeira colocação, com 346 citações, destaca-se o artigo de Jack \& Anderson (2002), que realizou um exame qualitativo das ações dos empresários rurais por meio da teoria da estrutura de Giddens.

\subsection{Análise de Cocitação}

$\mathrm{Na}$ análise de cocitações, é possível observar a frequência em que os autores são citados em conjunto em um mesmo artigo (Serra et al., 2012). Também é possível analisar grupos de autores que compõem o tema e características em comum.

Figura 2.2 - Mapa de Cocitações dos clusters nas cores Azul, Amarela, Vermelha e Verde.

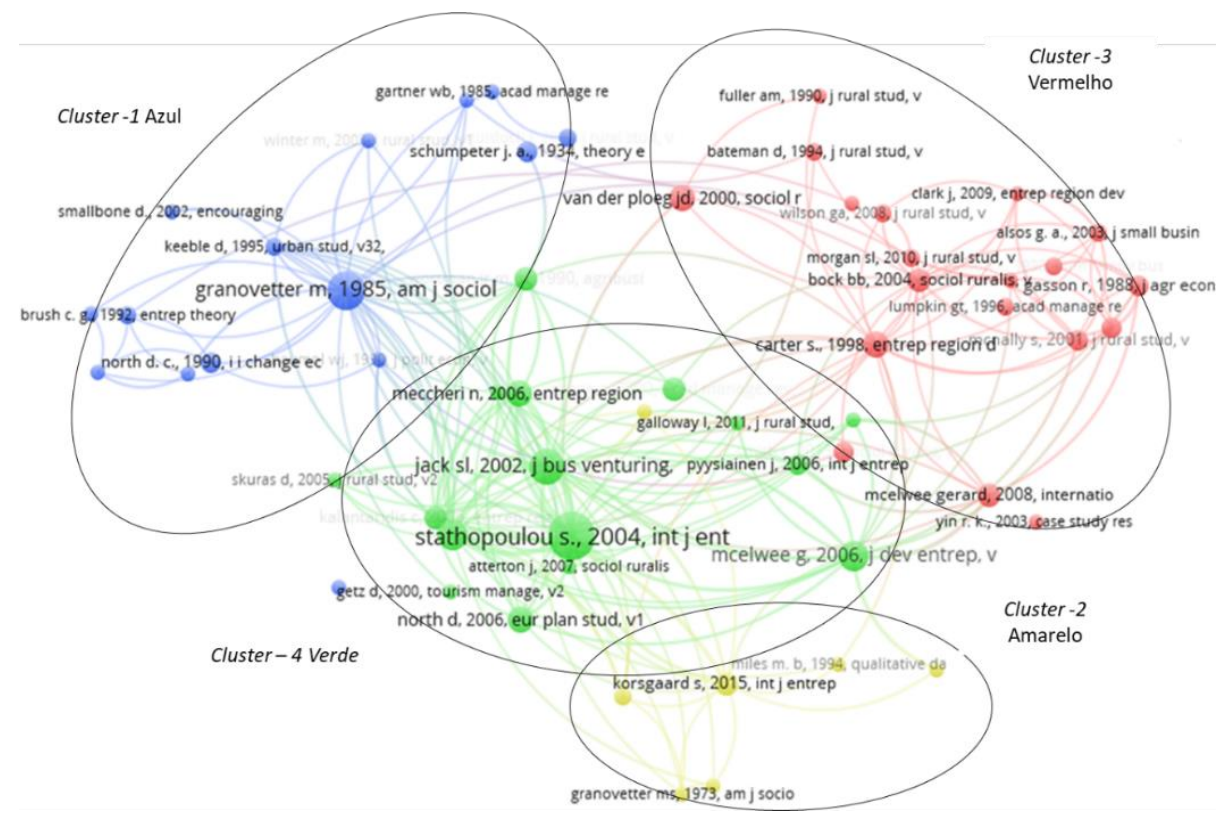

Fonte: Dados recolhidos do WOS e representação gráfica do software VOSviewer (2018) 
Os mapas de cocitações foram desenvolvidos por meio dos dados recolhidos do ISI Web of Science e realizados por meio do software VOSviewer. Foram criados 4 clusters, diferenciados com as cores azul, amarela, vermelha e verde. Cada cluster representa o agrupamento de pesquisadores que apresentam uma linha de pesquisa comum ou semelhante.

A análise realizada no cluster de cor Azul, é representado por artigos seminais de teorias. Como por exemplo, o artigo de Granovetter (1985), citado por muitos autores por analisar as relações entre o comportamento e instituições, artigo clássico da teoria social. Dentro outros seminais, que surgem no cluster Azul, temos Schumpeter (1934), com a Teoria do desenvolvimento econômico. Seminal citado por quase todos os artigos que tratam do tema empreendedorismo em seus diversos contextos e North (1990), que explora as características dos mercados políticos, caracterizando os custos de transação nos mercados políticos.

O Cluster de cor Amarela, são representados por pesquisas que analisam o fortalecimento dos recursos locais. Korsgaard \& Müller (2015), analisa o empreendedorismo rural por meio das atividades empresariais dos agricultores em contextos locais visando desenvolvimento.

Os artigos que representam o Cluster da cor verde, se destacam por analisar as habilidades e capacidade empresarial dos agricultores. Stathopoulou, Psaltopoulos, \& Skuras (2004), analisam as influências dinâmicas e complexas sobre as atividades empresariais nas áreas rurais. Jack \& Anderson (2002), descrevem a teoria de Giddens para desenvolver a concepção do empreendedorismo como processo socioeconômico integrado. Por fim, o papel e a função dos empresários rurais, suas habilidades e capacidade empresarial são analisadas sobre a ótica do desenvolvimento e do empreendedorismo em empresas no meio rural (G. McElwee, 2006; Nicola Meccheri \& Pelloni, 2006; D. North \& Smallbone, 2006).

Os artigos que representam o Cluster de cor vermelha, são formados por publicações que analisam as estratégias em seus processos de desenvolvimento, como é o caso do artigo de Van der Ploeg et al. (2000). Outras formas de estratégia são analisadas como alternativas de geração de renda por Carter (1998). Também é possível observar publicações que pesquisam fontes suplementares de renda "outras fontes" como forma estratégica de para o aumento da renda por agricultores de origem familiar (Bateman \& Ray, 1994).

\subsection{Análise Fatorial}

A análise fatorial é uma técnica de redução de dados que pode ser utilizada para determinar subcampos ou temas (Lin \& Cheng, 2010). Para a realização desta análise, a coleta de dados realizada na plataforma Web of Science foi analisada por meio do software Bibexcel que possibilitou a manipulação dos dados e utilização em formato de arquivo Excel para a realização da Análise Fatorial Exploratória no software IBM-SPSS v.22, resultados apresentados na Tabela 3.

Tabela 3 - Sumário da Análise de Fatores

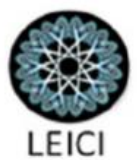




\begin{tabular}{|c|c|c|c|c|c|c|c|}
\hline \multicolumn{8}{|c|}{ Matriz de componente } \\
\hline \multicolumn{2}{|l|}{ FATOR (1) } & \multicolumn{2}{|c|}{ FATOR (2) } & \multicolumn{2}{|l|}{ FATOR (3) } & \multicolumn{2}{|c|}{ FATOR (4) } \\
\hline Autor & KMO & Autor & KMO & Autor & KMO & Autor & KMO \\
\hline AudretschD2004 &, 873 & Ajzenl1991 & 922 & WagnerJ2004 & 900 & GallowayL2011 & ,772 \\
\hline Terluinl2003 & 850 & SouitarisV2007 & ,910 & KingG2001 & 885 & BosworthG2012 & ,743 \\
\hline NorthD2000_A & ,848 & BirdB1988 & 907 & GibsonD2004 & 851 & DrigaO2009 & 682 \\
\hline NijkampP2003 & ,848 & WilsonF2007 & ,900 & KruegerJrN1994 & 840 & GetzD2000 & ,659 \\
\hline NorthD2000 & ,846 & LinanF2009 & ,898 & WennekersS1999 & ,838 & AttertonJ2007 & ,643 \\
\hline SkurasD2005 & ,842 & LinanF2007 & ,893 & SternbergR2005 & 807 & PezziniM2001 & ,580 \\
\hline KalantaridisC2006 & ,840 & FayolleA2006 & ,892 & SimonM2000 & ,639 & LafuenteE2007 & ,550 \\
\hline LabrianidisL2006 & ,838 & ZhaoH2005 & ,883 & ReynoldsP2005 & ,558 & & \\
\hline KalantaridisC2006_A & 815 & DohseD2012 & 873 & & & & \\
\hline DinisA2006 & ,812 & LangowitzN2007 & ,870 & & & & \\
\hline LordkipanidzeM2005 & 808 & KruegerN2000 & 868 & & & & \\
\hline AustinJ2006 & 794 & LinanF2011 & 843 & & & & \\
\hline FloraC1993 & ,789 & ChlostaS2012 & ,777 & & & & \\
\hline WestlundH2003 & ,783 & ColemanJ1988 & ,735 & & & & \\
\hline MeccheriN2006 & ,757 & & & & & & \\
\hline JackS2002 & ,733 & & & & & & \\
\hline StathopoulouS2004 & ,713 & & & & & & \\
\hline GranovetterM1973 & ,676 & & & & & & \\
\hline DavidssonP2003 & ,669 & & & & & & \\
\hline PyysiainenJ2006 & ,665 & & & & & & \\
\hline GranovetterM1985 & ,625 & & & & & & \\
\hline ShaneS2000 & ,610 & & & & & & \\
\hline NorthD2006 & ,597 & & & & & & \\
\hline BockB2004 & ,533 & & & & & & \\
\hline $\begin{array}{l}\text { \% Variância Total } \\
\text { Explicada }-73,96 \%\end{array}$ & 29,25 & & 21,90 & & 11,24 & & 11,57 \\
\hline
\end{tabular}

Fonte: Elaborado pelos autores

A Análise Fatorial foi realizada seguindo os procedimentos reconhecidos por Hair et al. (2005), que recomenda a avaliação do KMO (acima de 0,5) como indicador para identificar qual artigo pertence a qual fator, e dentre estes fatores, os fatores que representam um subcampo ou tema de possíveis pesquisa. Como pode ser observado na (Tabela 3), quatro fatores foram construídos, representando possivelmente quatro subcampos de pesquisa em Empreendedorismo Rural.

\section{Cluster 1 - Empreendedorismo Rural e o desenvolvimento no meio rural}

O grupo de autores do clusters 1, é representado por autores 24 artigos que o desenvolvimento no meio rural por meio do Empreendedorismo Rural, não diferente de outros estudos em empreendedorismo, o contexto rural atua como forma de estrutura no processo empreendedor e o empresário como agente dessa estrutura (Jack \& Anderson, 2002).

Contexto local analisado como desenvolvimento endógeno também foram considerados por pesquisados do Cluster 1, como forma de valores que impulsionam o 
desenvolvimento local (Labrianidis, 2006; Nijkamp, 2003; Terluin, 2003). Desenvolvimento exógenos, que diferente dos endógenos (não levam em consideração os valores locais), considerados como transplantados e vistos como dominantes foram tratados como importantes para serem analisados (Labrianidis, 2006). A principal mensagem deste grupo de autores, é o pertencimento ao local e o empreendedorismo endógeno utilizado como estratégia para o desenvolvimento rural Dinis (2006).

Outros fatores também podem ser encontrados para analisar tais empreendedores no contexto rural, como a capacidade de inovação como os novos negócios rurais, fazendas sustentáveis e turismo sustentável (Lordkipanidze, Brezet, \& Backman, 2005). Infraestrutura Social, que possibilita acesso a tecnologia como Internet e as rede de contatos que facilita acesso a captação de recurso, inovação e marketing (Dinis, 2006; Flora \& Flora, 1993; M. S. Granovetter, 1973).

A seguir, os artigos do Cluster 2, analisaram a intenção empreendedora e o comportamento empreendedor.

\section{Cluster 2 - Intenção Empreendedora e o Comportamento Empreendedor}

O segundo clusters, engloba autores que analisam capacidades e comportamento de agricultores como empresários no contexto rural, representados por 14 autores, conforme tabela 3 .

O artigo seminal de Ajzen (1991), se destaca no grupo por ser referência principal entre os demais artigos que utilizaram a teoria para analisarem os seus artigos, o autor sugere em seu estudo um modelo de intenções, para análise do comportamento planejado. Estudo que utilizaram os modelos foram realizados para análise de fatores motivacionais (Liñán, Urbano, \& Guerrero, 2011).

Intenções Empreendedoras visam a criação de um novo negócio ou novos valores ao negócio existe (Bird, 1988). Situações que dependem da condição emocional e financeira do empreendedor (Krueger, Reilly, \& Carsrud, 2000). As Intenções Empreendedoras, também podem ser avaliadas em termos de capacidade, por meio da Teoria do Comportamento Planejadom(TPB) de Ajzen, como já apresentado anteriormente ou pelo modelo de Shapero do Evento Empreendedor (SEE) (Chlosta, Patzelt, Klein, \& Dormann, 2012).

\section{Cluster 3 - Empreendedorismo e Crescimento Econômico}

No cluster 3, com 08 artigos, são representados por artigos que analisam o crescimento econômico por meio do empreendedorismo.

Crescimento Econômico é tema de extrema importância e por isso é estudado e analisado em diferentes contextos. A Organização para a Cooperação e Desenvolvimento Econômico (OCDE), fornece plataforma para comparar o desenvolvimento econômico em diferentes países (King \& Zeng, 2001). O programa e pesquisa Global Entrepreneurship

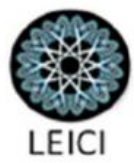


Monitor (GEM), também concentra suas pesquisas no crescimento econômico, mas é utilizada como uma avaliação do papel do empreendedorismo em tal crescimento. Com o uso de ferramentas de pesquisas, é possível indicar atividades empresariais, renda das famílias, escolaridade e diversos outros temas que podem ser utilizados como fontes de informações.

Dentre os artigos do cluster 3, também se destacam estudos sobre desenvolvimento individual de indivíduos (Gibson, 2004). Atividades Empreendedoras (Wagner \& Sternberg, 2004). Fatores de risco por indivíduos, por não perceberem o risco ao iniciar um novo negócio e/ou atividade empreendedora (Simon, Houghton, \& Aquino, 2000) e por fim, potencial empreendedor baseado no modelo de (Krueger \& Deborah Brazeal, 1994; Shapero \& Sokol, 1982).

\section{Clusters 4 - Foco Empresarial dos Empreendedores Rurais}

O clusters 4, representado por 7 artigos, é caracterizado por grupo de autores que analisam o comportamento empresarial dos empreendedores rurais.

Lafuente, Vaillant \& Rialp (2007), analisaram a influência de modelos empresariais em atividades empresariais no contexto rural. Famílias de empreendedores rurais buscam no setor inovar para buscar novas formas de desenvolvimento, como negócios voltados para o turismo, como as fazendas que oferecem conforto de hotel e convívio com o meio ambiente para seus hospedes (Getz \& Carlsen, 2000). Em um ambiente rural, as empresas familiares necessitam do seu ambiente para promover seu negócio e se utiliza de laços sociais para realizar essa interação, se o empreender é um empresário nascente (que nasceu naquela região), essa ligação acaba se tornando mais forte (Gerard McElwee \& Bosworth, 2010). Tais redes, também possibilita acesso à Internet e acesso a comercialização por meio da comercialização pelos meios de comunicação disponível na região (Bosworth, 2012).

Com uso da análise fatorial, foi possível observar quatro campos distintos consolidados para estudos em empreendedorismo rural e com o uso da análise cocitações, ma.pa representando grupos de autores em redes, identificamos co-ocorrência (grupo de autores) que publicam no tema Empreendedorismo Rural. Analises essas que contribuíram para identificação do campo de pesquisa.

\section{Considerações Finais}

O estudo teve como objetivo realizar análise do panorama das publicações sobre empreendedorismo rural por meio de um estudo bibliométrico. $\mathrm{Na}$ contextualização da produção científica, os resultados apresentaram uma crescente diversidade de publicações sobre o tema possibilitando identificar os autores e periódicos que publicam.

Os resultados obtidos identificam a formação de quatro cluster de autores que trabalham com o tema empreendedorismo rural em diferentes contextos, mas com foco no desenvolvimento local. Dentre os estudos analisados, vale reforçar (Alemu \& Adesina, 2017),
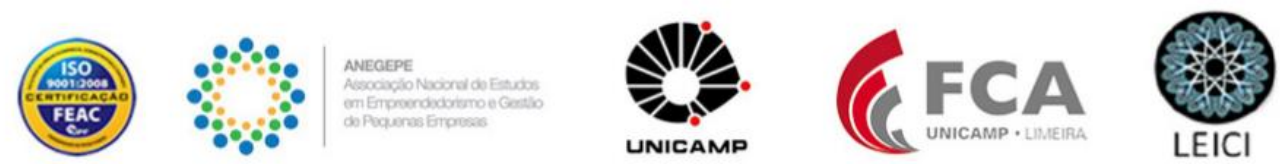
destacando a importância da transformação agrária como fator crucial para mudar as condições de vida dos moradores rurais nos países em desenvolvimento. Van der Ploeg et al., (2000), no que diz respeito ao desenvolvimento no meio rural. O autor acredita que muitos cientistas ainda possuem resistência quanto ao desenvolvimento rural que veem emergindo lentamente em uma perspectiva de mudança de paradigma.

Também foi possível observar grupos de pesquisadores que remetem o empreendedorismo rural a desenvolvimentos econômicos com embasamento na teoria econômica de Schumpeter (1934), que possibilita a associações ao uso das temáticas de inovação no meio rural, como o desenvolvimento do agricultor familiar, características do empreendedor no meio rural, habilidades empresariais dos agricultores, orientação empreendedora, influência do contexto, redes de relacionamentos entre outras diversas linhas de pesquisa que relacionam o empreendedorismo com o desenvolvimento do meio rural.

As pesquisas apontam que o campo de pesquisa está em ascensão e o uso do termo "empreendedorismo rural" vem crescendo no meio acadêmico e se consolidando, demonstrando uma possível evolução científica de estudos no meio rural e definição de novas agendas de pesquisas para à área. Para estudos futuros, sugere-se realizar analisar o comportamento e habilidades do empreendedor em um contexto tão difícil quanto o contexto rural e estudo específicos nos principais journals e congressos é recomendado.

\section{Referências}

Abramovay, R., Magalhães, R., \& Schroder, M. (2010). Representatividade e inovação na governança dos processos participativos: o caso das organizações Brasileiras de agricultores familiares. Sociologias, 12(24), 268-306.

Alemu, A. E., \& Adesina, J. O. (2017). In Search of Rural Entrepreneurship: Non-farm Household Enterprises (NFEs) as Instruments of Rural Transformation in Ethiopia. African Development Review, 29(2).

Anderson, A. R. (2000). Paradox in the periphery: An entrepreneurial reconstruction? Entrepreneurship and Regional Development, 12(2), 91-109.

Ateljevic, I., \& Doorne, S. (2000). "Staying within the fence": Lifestyle entrepreneurship in tourism? Journal of Sustainable Tourism, 8(5), 378-392.

Barreto, J. C., \& Nassif, V. M. J. (2014). O Empreendedor Líder e a Disseminação da Orientação Empreendedora. Revista Brasileira de Gestao de Negocios, 16(51), 180-198.

Bateman, D., \& Ray, C. (1994). Farm pluriactivity and rural policy: Some evidence from Wales1. Journal of Rural Studies, 10(1), 1-13.

Bock, B. B. (2004). Fitting in and multi-tasking: Dutch farm women's strategies in rural entrepreneurship. Sociologia Ruralis, 44(3).

Bosworth, G., Dana, L.-P., \& McElwee, G. (2011). EU industrial policy and competitiveness in rural SMEs. International Journal of Entrepreneurship and Small Business, 14(3).

Carter, S. (1998). Portfolio entrepreneurship in the farm sector: Indigenous growth in rural areas? Entrepreneurship and Regional Development, 10(1), 17-32.

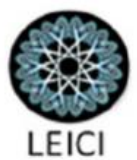


Clark, J. (2009). Entrepreneurship and diversification on English farms: Identifying business enterprise characteristics and change processes. Entrepreneurship and Regional Development, 21(2), 213-236.

Dinis, A. (2006). Marketing and innovation: Useful tools for competitiveness in rural and peripheral areas. European Planning Studies, 14(1), 9-22. 3.

Driga, O., Lafuente, E., \& Vaillant, Y. (2009). Reasons for the relatively lower entrepreneurial activity levels of rural women in Spain. Sociologia Ruralis, 49(1), 70-96.

Esparcia, J., Escribano, J., \& Serrano, J. J. (2015). From development to power relations and territorial governance: Increasing the leadership role of LEADER Local Action Groups in Spain. Journal of Rural Studies, 42, 29-42.

FAO. (2014). Ano Internacional da Agricultura Familiar 2014. Retrieved from http://www.fao.org/family-farming-2014/home/what-is-family-farming/pt/

Ferreira, M. P., Reis, N. R., \& Miranda, R. (2015). Thirty years of entrepreneurship research published in top journals: analysis of citations, co-citations and themes. Journal of Global Entrepreneurship Research, 3(3), 205-209.

Figueroa-armijos, M., \& Johnson, T. G. (2013). Entrepreneurship in Rural America Across, 18(2), 138.

Galloway, L., Sanders, J., \& Deakins, D. (2011). Rural small firms' use of the internet: From global to local. Journal of Rural Studies, 27(3), 254-262.

Getz, D., \& Carlsen, J. (2000). Characteristics and goals of family and owner-operated businesses in the rural tourism and hospitality sectors. Tourism Management, 21(6), 547-560.

Gibson, D. E. (2004). Role models in career development: New directions for theory and research. Journal of Vocational Behavior, 65(1), 134-156.

Gnyawali, D. R., \& Fogel, D. S. (1994). Environments for entrepreneurship development: key dimensions and research implications. Entrepreneurship: Theory \& Practice, 18(August), 43-62.

Granovetter, M. (1985). Economic action and social structure: The problem of embeddedness. American Journal of Sociology, 91(3), 481-510.

Granovetter, M. S. (1973). The strength of weak ties. In Social Networks, (pp. 347-367).

Grimes, S. (2000). Rural areas in the information society: Diminishing distance or increasing learning capacity? Journal of Rural Studies, 16(1), 13-21.

Hair, J. F., Black, W. C., Babin, B. J., Anderson, R. E., \& Tatham, R. L. (2009). Análise multivariada de dados.

Hair, J. F. (2005). Livro_ANALISE MULTIVARIADA DE DADOS - Joseph Hair2005.pdf.

Henry, C., \& McElwee, G. (2014). Defining and conceptualising rural enterprise. Contemporary Issues in Entrepreneurship Research (Vol. 4).

Hindle, K., \& Moroz, P. (2010). Indigenous entrepreneurship as a research field: Developing a definitional framework from the emerging canon. International Entrepreneurship and Management Journal, 6(4), 357-385.

Jack, S. L., \& Anderson, A. R. (2002). The effects of embeddedness on the entrepreneurial process. Journal of Business Venturing, 17(5).

Kalantaridis, C., \& Bika, Z. (2006). Local embeddedness and rural entrepreneurship: Case-study evidence from Cumbria, England. Environment and Planning A, 38(8), 1561-1579. 
Korsgaard, S., \& Müller, S. (2015). Rural entrepreneurship or entrepreneurship in the rural - between place and space. International Journal of Entrepreneurial Behaviour and Research, 21(1).

Lafuente, E., Vaillant, Y., \& Rialp, J. (2007). Regional differences in the influence of role models: Comparing the entrepreneurial process of rural Catalonia. Regional Studies, 41(6).

Lamarche, H. (1993). A agricultura familiar: comparaçao internacional: Uma realidade multiforme. Tradução de Ângela Maria Naoko Tijiwa. Campinas - SP, 11-33.

Lin, T.-Y., \& Cheng, Y.-Y. (2010). Exploring the Knowledge Network of Strategic Alliance Research: A Co-Citation Analysis. International Journal of Electronic Business Management, 8(2), 152 160.

McElwee, G. (2006). The enterprising farmer: A review of entrepreneurship in agriculture. Journal of the Royal Agricultural Society of England, 167.

McElwee, G. (2008). A taxonomy of entrepreneurial farmers. International Journal of Entrepreneurship and Small Business, 6(3), 465.

Meccheri, N., \& Pelloni, G. (2006). Rural entrepreneurs and institutional assistance: An empirical study from mountainous Italy. Entrepreneurship and Regional Development, 18(5), 371-392.

Niska, M., Vesala, H. T., \& Vesala, K. M. (2012). Peasantry and Entrepreneurship As Frames for Farming: Reflections on Farmers' Values and Agricultural Policy Discourses. Sociologia Ruralis, 52(4), 453-469.

North, D. C. (1990). A Transaction Cost Theory of Politics. Journal of Theoretical Politics, 2(4), 355367.

North, D., \& Smallbone, D. (2006). Developing entrepreneurship and enterprise in Europe's peripheral rural areas: Some issues facing policy-makers. European Planning Studies, 14(1).

Pato, L. (2015). Rural entrepreneurship and Innovation: some sucessful women's initiatives.

Pato, M. L., \& Teixeira, A. A. C. (2016). Twenty Years of Rural Entrepreneurship: A Bibliometric Survey. Sociologia Ruralis, 56(1).

Pinto, R. F., Guerrazzi, L. de C., Serra, B. de C., \& Kniess, C. T. (2016). A Pesquisa em Administração Estratégica: Um Estudo Bibliométrico em Periódicos Internacionais de Estratégia no Período de 2008 A 2013. Revista Ibero-Americana de Estratégia, 15(2), 22-37.

Reynolds, P., Bosma, N., Autio, E., Hunt, S., De Bono, N., Servais, I., ... Chin, N. (2005). Global entrepreneurship monitor: Data collection design and implementation 1998-2003. Small Business Economics, 24(3), 205-231.

Schumpeter, J. A. (1934). The theory of economic development (Cambridge.).

Schumpeter, J. A. (1982). The Theory of Economic Development: An Inquiry into Profits, Capital, Credit, Interest, and the Business Cycle (1912/1934).

Serra, F. R., Ferreira, M. P., de Almeida, M. I. R., \& de Souza Vanz, S. A. (2012). A pesquisa em administração estratégica nos primeiros anos do século XXI: Um estudo bibliométrico de citações e co-citações no Strategic Management Journal entre 2001 e 2007. Estratégia \& Negócios, v.5, n.2, 257-274.

Shane, S., \& Venkataraman, S. (2000). The promise of entrepreneurship as a field of research. Academy of Management Review, 25(1), 217-226.

Smith, R., \& McElwee, G. (2015). Developing qualitative research streams relating to illegal rural enterprise reflections on researching qualitatively at the margins of entrepreneurship research.

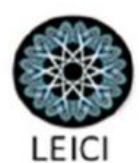


International Journal of Entrepreneurial Behaviour and Research, 21(3).

Stathopoulou, S., Psaltopoulos, D., \& Skuras, D. (2004). Rural entrepreneurship in Europe. International Journal of Entrepreneurial Behavior \& Research, 10(6), 404-425.

Steyaert, C., \& Katz, J. (2004). Reclaiming the space of entrepreneurship in society: Geographical, discursive and social dimensions. Entrepreneurship and Regional Development, 16(3), 179-196.

Terluin, I. J. (2003). Differences in economic development in rural regions of advanced countries: An overview and critical analysis of theories. Journal of Rural Studies, 19(3), 327-344

Vaillant, Y., \& Lafuente, E. (2007). Do different institutional frameworks condition the influence of local fear of failure and entrepreneurial examples over entrepreneurial activity? Entrepreneurship and Regional Development, 19(4), 313-337.

Van der Ploeg, J. D., Renting, H., Brunori, G., Knickel, K., Mannion, J., Marsden, T., ... Ventura, F. (2000). Rural Development: From Practices and Policies towards Theory. Sociologia Ruralis, 40(4), 391-408.

Van Eck, N. J. \& Waltman, L. (2014). Manual for VOSviewer Version 1.5.7.

Veiga, J. E. Da. (2001). O Brasil rural ainda não encontrou seu eixo de desenvolvimento. Estudos Avançados, 15(43), 101-119.

Venkataraman, H., Vermeulen, P., Raaijmakers, A., \& Mair, J. (2016). Market Meets Community: Institutional Logics as Strategic Resources for Development Work. Organization Studies, 37(5), 709-733.

Venkataraman, S. (1997). The Distinctive Domain of Entrepreneurship Research. Advances in Entrepreneurship, Firm Emergence and Growth, 3(October), 119-138.

Wanderley, M. D. (1998). O Brasil: agricultura familiar ou latifúndio. LAMARCHE, HA A Agricultura Familiar., 2(Campinas, Ed Unicamp), 27-31.

Wanderley, M. D. N. B. (2003). Agricultura familiar e campesinato: rupturas e continuidade. Estudos Sociedade E Agricultura, outubro(21), 20.

Wortman, M. S. (1990). A unified approach for developing rural entrepreneurship in the US. Agribusiness, 6(3).

Zanin, L. M., \& Silva, F. R. (2015). Evolução das Teorias que suportam os Artigos Publicados em Empreendedorismo entre 1960 e 2013: Análise da rede de citação e co-citação. XXXIX Encontro Do ANPAD, (September 2015), 1-20. 